\title{
Pigmentation Effect of Rice Bran Extracted Minerals Comprising Soluble Silicic Acids
}

\author{
Hyun-Jun Jang and Young-Kwon Seo \\ Department of Medical Biotechnology (BK21 Plus team), Dongguk University, Seoul, Republic of Korea \\ Correspondence should be addressed to Young-Kwon Seo; bioseo@dongguk.edu
}

Received 28 July 2016; Revised 27 September 2016; Accepted 11 October 2016

Academic Editor: Gioacchino Calapai

Copyright ( $) 2016$ H.-J. Jang and Y.-K. Seo. This is an open access article distributed under the Creative Commons Attribution License, which permits unrestricted use, distribution, and reproduction in any medium, provided the original work is properly cited.

\begin{abstract}
Our investigation focused on identifying melanogenesis effect of soluble minerals in rice bran ash extract (RBE) which include orthosilicic acid (OSA). Melanocytes were apparently normal in terms of morphology. It was, however, shown that they were stressed a little in the RBE and OSA added media in aspect of LDH activity. Melanin synthesis and intracellular tyrosinase activity were increased by treatment of RBE which is similar to that of OSA. The Western blotting results showed that TRP-1, tyrosinase, and MITF expression levels were 2-3 times higher in the OSA and RBE groups compared to the control group which promoted melanin synthesis through CREB phosphorylation. Moreover, histology and immunohistochemistry were shown to have similar result to that of protein expression. As a result, minerals which comprise orthosilicic acid has the potential to promote melanogenesis and both RBE and OSA have similar cell viability, protein expression, and immunostaining results, suggesting that RBE comprises specific minerals which promote melanin synthesis through increasing of MITF and CREB phosphorylation. Therefore, RBE could be used as a novel therapeutic approach to combat melanin deficiency related diseases by stimulating melanocytes via its soluble $\mathrm{Si}$ and mineral components.
\end{abstract}

\section{Introduction}

Melanin deficiency diseases, such as canities and vitiligo, primarily result from dysfunction of the melanocytes or cell aging; thus many therapies to treat and/or stop the progression of melanin deficiency diseases have been investigated.

Numerous studies have revealed that various natural extracts promote melanocyte activation. Hong et al. reported that black soybean extracts have melanogenic effects in Melan-a cells, which increase melanin contents and intracellular tyrosinase activity approximately 30 and $20 \%$, respectively [1]. Similarly, An et al. investigated the melanogenesis effect of Cornus officinalis extract in Melan-a cells. Cornus officinalis extract-treated cells had $36.1 \%$ more melanin content and upregulated expression of tyrosinase, tyrosinase-related protein 1 (TRP-1) and 2 (TRP-2), and microphthalmia-associated transcription factor (MITF) [2]. Furthermore, Park et al. showed that the extract of Zingiber cassumunar Roxb. had a melanogenesis effect in B16F10 melanoma cells. The components enhanced extracellular signal-regulated kinase (ERK) activation, tyrosinase activity, and upstream stimulating factor-1 (USF1) levels. Additionally, melanin synthesis was decreased in USF1-knockdown cells [3].

Similar to these above-mentioned studies, many researchers have used organic materials to stimulate melanogenesis. However, to the best of our knowledge, the use of inorganic mineral components to stimulate melanocytes has not been published. Rice bran ash extract (RBE) has had its organic compounds removed by incineration, leaving organic compounds, such as $\mathrm{Si}, \mathrm{K}, \mathrm{P}$, and soluble silicic acids.

Silicic acid exists in various forms, including a family of compounds with the formula $\left[\mathrm{SiO}_{x}(\mathrm{OH})_{4-2 x}\right]_{n}$, such as silicon dioxide $\left(\mathrm{SiO}_{2}\right)$. Most of these compounds are waterinsoluble, causing cytotoxicity in vivo. Among them, however, orthosilicic acid $\left(\mathrm{OSA}, \mathrm{Si}(\mathrm{OH})_{4}\right)(\mathrm{OSA})$ is water-soluble and has low toxicity compared to insoluble silicic acids $[4,5]$.

The effects of OSA have been investigated in various cell types by numerous researchers. Reffitt et al. reported OSA promoted collagen 1 synthesis and increased the expression 
of alkaline phosphatase and osteocalcin in osteosarcoma cell line MG-63 [6,7]. In an aged, ovariectomized rat, OSA supplementation increased femoral and lumbar bone mineral density by decreasing $\mathrm{Ca}$ excretion and slowing down bone turnover [8]. Also, the combination of OSA and $\mathrm{Ca} /$ vitamin D3 increased bone mineral density compared to $\mathrm{Ca}$ /vitamin D3 alone in a double-blind placebocontrolled clinical study [9], suggesting that OSA shows potential as a new treatment for bone-related diseases, such as osteoporosis.

In addition to its benefits in bone-related diseases, Calomme and Vanden Berghe reported that OSA intake increased collagen concentration by approximately $10 \%$ in dermis and cartilage [10], suggesting that Si maybe involved in both extracellular matrix formation and Ca metabolism. Also, Grotheer et al. showed that OSA had anti-inflammatory properties in chronic wounds [11] and Barel et al. reported that the positive effects of OSA, on skin, hair, and nails, increased with the increase in hydroxyproline concentration in the dermis [12].

Whilst the use of natural materials to promote bone, skin, and cartilage repair has been extensively studied and the organic compounds and minerals from RBE have been used for cell activation, to the best of our knowledge, no studies have been published on the use of mineral components to stimulate melanocytes or promote melanogenesis. Therefore, this study investigated the pigmentation-promoting effect of OSA and the combination of OSA with RBE, which contains mineral components, such as soluble Si.

\section{Materials and Methods}

2.1. Material. RBE was obtained from the carbonized chaff of rice bran. Rice bran ash $(200 \mathrm{~g})$ was added to $1 \mathrm{~L}$ of distilled water and stirred at $400 \mathrm{rpm}$ and $100^{\circ} \mathrm{C}$ for $24 \mathrm{~h}$. After that, the mixture was filtered through $10 \mu \mathrm{m}$ filter paper, centrifuged at $10000 \mathrm{rpm}$ at $25^{\circ} \mathrm{C}$ for $30 \mathrm{~min}$ to remove any remaining particulate matter, and then sterilized using a $0.2 \mu \mathrm{m}$ syringe filter. OSA was obtained from Natural Factors, BioSil, USA.

2.2. Cell Culture. QualiCell human melanoblasts (Creative Bioarray, New York, USA) were cultured in medium 254 (M254, Invitrogen, Waltham, USA) containing PMA-free human melanocyte growth supplement (HMGS-2, Invitrogen, Waltham, USA), 50 units $/ \mathrm{mL}$ penicillin, and $50 \mu \mathrm{g} / \mathrm{mL}$ streptomycin (HyClone, USA) by incubation in an $80 \mathrm{~mm}$ dish at $37^{\circ} \mathrm{C}$ in a humidified atmosphere with $5 \% \mathrm{CO}_{2}$.

2.3. Melanin Content Determination. Melanoblasts were seeded at $1 \times 10^{4}$ cells/well into 6-well plates and cultured for 3 days in differentiation media containing HMGS-2, $10 \mathrm{nM} \alpha$-melanocyte-stimulating hormone $(\alpha$-MSH, SigmaAldrich), 10 nM 12-O-tetradecanoylphorbol-13-acetate (TPA, Sigma-Aldrich), and $20 \mu \mathrm{M}$ forskolin (Sigma-Aldrich). Then, the media was exchanged for M254 media containing $0.24 \mu \mathrm{g} / \mathrm{mL}$ OSA and $30 \mu \mathrm{g} / \mathrm{mL} \mathrm{RBE}$ and the differentiated melanocytes were cultured for further 3 days.
The melanin contents in melanocytes were determined using a previously published method [13] with modifications. After removing the culture media from the plates, the cells were lysed with $10 \%$ DMSO solution, dissolved in $1 \mathrm{M} \mathrm{NaOH}$, and boiled at $80^{\circ} \mathrm{C}$ for $2 \mathrm{~h}$. Then, the cells were centrifuged at $15000 \mathrm{rpm}$ for $15 \mathrm{~min}$. The melanin content of the supernatant was measured using an ELISA plate reader at $405 \mathrm{~nm}$ (Spectrum Analyzer, Victor 1420-050, PerkinElmer Life Sciences, Turku, Finland).

2.4. Tyrosinase Activity Determination. Intracellular tyrosinase activity was measured using a previously described method [13] with slight modifications. Melanoblasts were seeded at $1 \times 10^{4}$ cells/well in 6-well plates and cultured for 3 days in differentiation media. Then, the medium was exchanged for M254 containing $0.24 \mu \mathrm{g} / \mathrm{mL}$ OSA and $30 \mu \mathrm{g} / \mathrm{mL} \mathrm{RBE}$ and the differentiated melanocytes were cultured for further 3 days. After removing the culture media from the plates, the cells were washed with PBS and lysed with $10 \%$ triton X-100 (Sigma-Aldrich). The cells were centrifuged at $15000 \mathrm{rpm}$ for $15 \mathrm{~min}$, and then the protein content of the supernatants was measured by the bicinchoninic assay (BCA) (Thermo Fisher Scientific, USA). 3,4,Dihydroxyphenylalanine (L-DOPA) (Sigma-Aldrich) (10\%) was dissolved in sodium phosphate buffer $(10 \mathrm{mM})$. After 30 min incubation at $37^{\circ} \mathrm{C}$, absorbance was measured at $475 \mathrm{~nm}$ using an ELISA plate reader (PerkinElmer Life Sciences).

2.5. Cell Damage Evaluation. Cell damage was evaluated by the lactate dehydrogenase $(\mathrm{LDH})$ assay. Aliquots $(100 \mu \mathrm{L})$ of the media obtained after culturing the cells for 3 days in M254 containing OSA and RBE (above-mentioned) were placed in a 96-well plate, $50 \mu \mathrm{L}$ of an LDH-LQ kit solution (Asan Pharmaceutical Inc., Korea) was added, and then the plates were incubated at $25^{\circ} \mathrm{C}$ for $30 \mathrm{~min}$. The reaction was stopped by adding $50 \mu \mathrm{L} 1 \mathrm{~N} \mathrm{HCl}$ and then absorbance was measured at $490 \mathrm{~nm}$.

2.6. Western Blotting. Human-derived melanoblasts were seeded in $60 \mathrm{~mm}$ dishes $(N=3)$ at a density of $1 \times 10^{5}$ cells and incubated for 3 days. The medium was removed, and the cells were washed twice with phosphate-buffered saline (PBS) and lysed in PBS containing 10\% glycerol, 5\% $\beta$ mercaptoethanol, $2 \%$ sodium dodecyl sulfate (SDS), and $0.01 \%$ bromophenol blue in a $62.6 \mathrm{mM}$ Tris- $\mathrm{HCl}$ buffer $(\mathrm{pH}$ 6.8). The cell lysates were then denatured at $100^{\circ} \mathrm{C}$ for $5 \mathrm{~min}$. The protein content of the cell lysates was quantified by the BCA assay, and equal amounts of protein per sample were separated by $10 \%$ SDS-polyacrylamide and electrotransferred onto a nitrocellulose membrane (Millipore Co., Massachusetts). The membranes were blocked in 5\% fat-free skim milk dissolved in Tris-buffered saline (TBS) containing $0.1 \%$ Tween 20 (TBS-T buffer) at room temperature for 1h. After washing with TBS-T, the membrane was incubated for 1 hour in 10\% bovine serum albumin, containing primary antibodies: anti-mouse $\beta$-actin antibody $(1: 2000)$, anti-goat TRP-1 antibody (1:500), anti-goat tyrosinase 


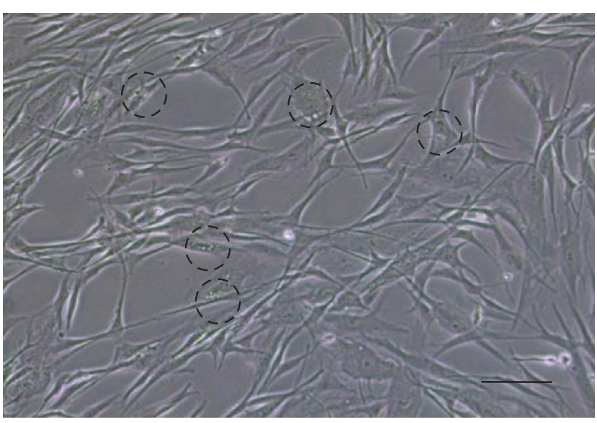

(a)

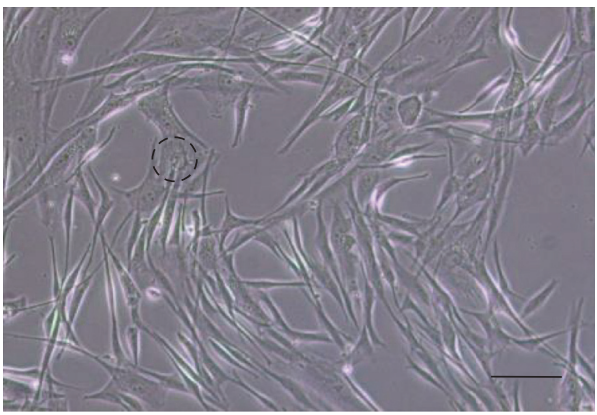

(c)

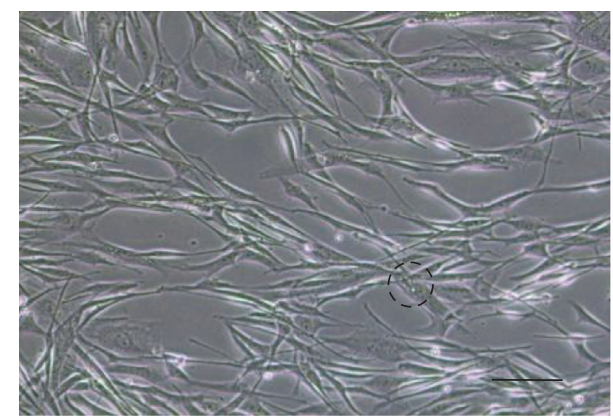

(b)

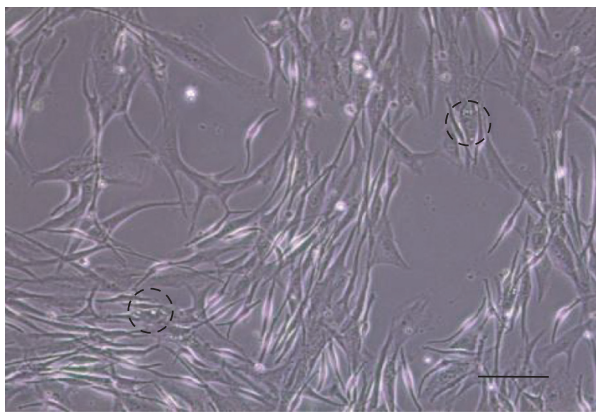

(d)

FIGURE 1: Representative images of melanocytes after 3 days cultured in differentiation media. (a) Control, (b) $10 \mathrm{nMSH}$, (c) $2.4 \mu \mathrm{g} / \mathrm{mL}$ OSA, and (d) $30 \mu \mathrm{g} / \mathrm{mL}$ RBE. Original magnification: (a) $-(\mathrm{d}) \times 100$; scale bar: $100 \mu \mathrm{m}$.

antibody (1:500), anti-rabbit MITF antibody (1:500), antirabbit CREB (cAMP-responsive element binding protein) antibody $(1: 1000)$, and anti-rabbit P-CREB (phosphorylatedCREB) antibody $(1: 1000)$. The primary antibodies were removed; bound primary antibody was detected by applying a horseradish peroxidase- (HRP-) conjugated anti-rabbit, antigoat, and anti-mouse secondary antibody for $2 \mathrm{~h}$ at room temperature. The membrane was washed in TBS-T; the blot was visualized with enhanced chemiluminescence reagent (Thermo Fisher Scientific, USA) and photographed using a gel imaging system, ChemiDoc XRS+ (Bio-Rad, Hercules, CA, USA). The results were quantified using ImageJ software (National Institutes of Health, Bethesda, MD, USA).

2.7. Immunohistochemistry. Immunohistochemistry was conducted after culturing the cells for 3 days in M254 media containing OSA and RBE (above-mentioned). Monolayer cultured melanocytes were fixed in $4 \%$ buffered formaldehyde solution and then incubated with anti-melanoma monoclonal antibody HMB45 solution (1:1000). Localization of HMB45 was performed through an avidinimmunoalkaline phosphatase method with vector red (Vector Laboratories, Burlingame, CA), which is a red chromogen product critical to the success of immunolocalization in pigmented tissues. Microscopic images were captured with a Nikon digital camera attached to a Nikon Optiphot-2 microscope [14].

2.8. Histochemical Evaluation. The histochemical evaluation was conducted after culturing the cells for 3 days in M254 media containing OSA and RBE (above-mentioned). The samples were fixed in $4 \%$ paraformaldehyde, sectioned, and stained with Fontana-Masson silver nitrate (Kojima Chemical, Kashiwabara, Japan) for $1 \mathrm{~h}$ at $56^{\circ} \mathrm{C}$ and then washed with distilled water and fixed in 5\% sodium thiosulfate solution (Duksan, Seoul, Korea) for $5 \mathrm{~min}$ and washed with distilled water. After that, samples were stained with nuclear fast red solution (Fluka, Buchs, Switzerland) for $5 \mathrm{~min}$ and washed with distilled water 3 times. Finally, the samples were dehydrated with $95 \%$ and then $100 \%$ ethanol and then washed with xylene (Duksan) 2 times [14].

\section{Results}

3.1. Evaluation of Cell Damage. Compared to the cells in the MSH, OSA, and RBE, those in the control group showed a greater number of vacuoles in the cytoplasm, indicating that these cells had aged more (Figure 1(a), dot circle). Furthermore, the MSH, OSA, and RBE cells showed more bipolar dendritic processes than the control cells and no apoptotic or necrotic cell death (Figure 1). However, OSA and RBE cells had $10 \%$ greater LDH activity, compared to the control group (Figure 2), indicating cell stress.

3.2. Melanin Contents and Tyrosinase Activity. Melanin levels were $10 \%$ higher in the $\mathrm{MSH}$, OSA, and RBE groups compared to the control group (Figure 3). Also, tyrosinase activity was approximately $10 \%$ higher in the OSA and MSH groups and $20 \%$ higher in the RBE group compared to the control group (Figure 4). 


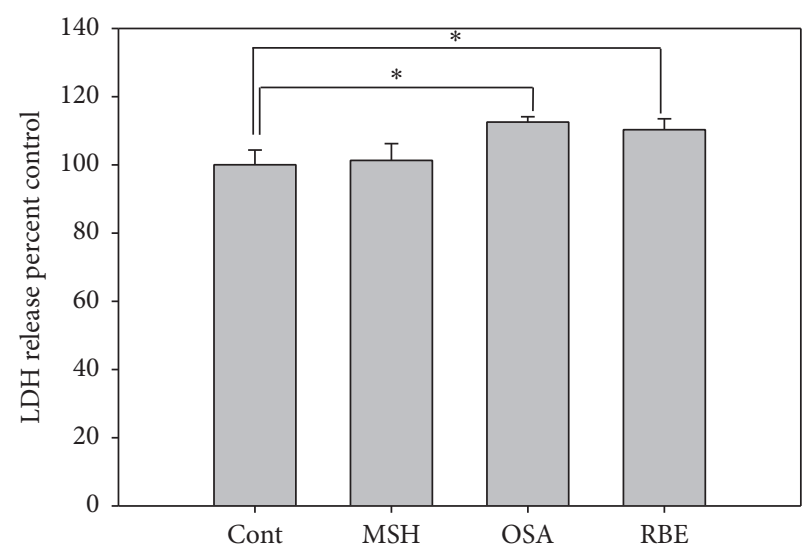

FIGURE 2: Representative graph of the LDH assay in melanocytes after 3 days cultured in differentiation media and after 3 days cultured in MSH, OSA, and RBE enriched media. Significant differences were determined by Student's $t$-test; ${ }^{*} P<0.05$.

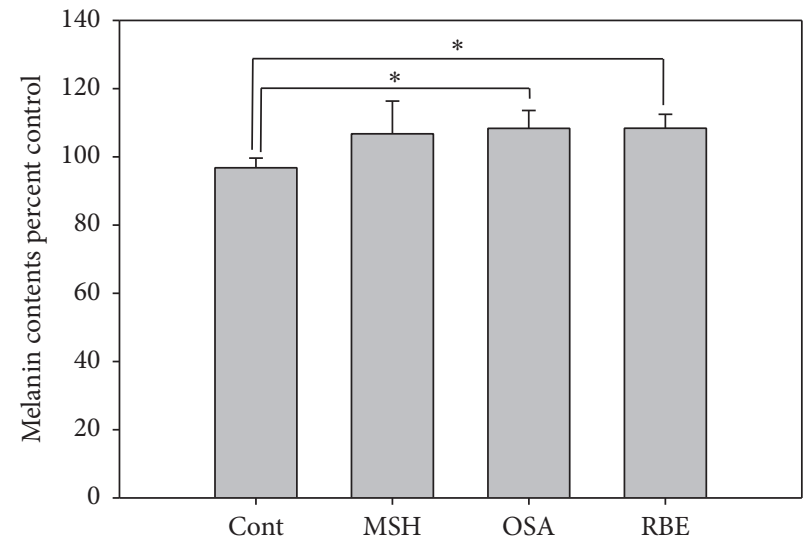

FIGURE 3: Representative graph of melanin contents in melanocytes after 3 days cultured in differentiation media and after 3 days cultured in MSH, OSA, and RBE enriched media. Significant differences were determined by Student's $t$-test; ${ }^{*} P<0.05$.

3.3. Protein Expression Levels. Protein expression of the melanogenesis markers TRP-1, tyrosinase, and MITF was assessed by Western blotting. As shown in Figure 5, TRP-1 and MITF increased 2.5-fold in the RBE group and 2-fold in the OSA group compared to the control. Tyrosinase increased 2 -fold and 1.5-fold in the RBE and OSA group, respectively, compared to the control. This result suggested that OSA and MSH had comparable efficacy to stimulate melanogenesis, whilst RBE had greater efficacy than MSH. Furthermore, the $\mathrm{P}$-CREB level increased approximately 2-fold in the MSH, OSA, and RBE groups, suggesting that melanin synthesis occurred via CREB phosphorylation.

3.4. Histological Evaluation and Immunohistochemistry. Fontana-Masson staining is used to confirm the fact that melanocytes are synthesizing melanin [15]. In this study, $\mathrm{MSH}, \mathrm{OSA}$, and RBE groups were positive for FontanaMasson staining, whilst the control group was negative (Figure 6). Similarly, MSH, OSA, and RBE groups were positive for HMB45, whilst the control group was negative (Figure 7) (Table 2). HMB45 is targeted to the melanosome [16]. Localized HMB45 staining indicates active melanosome formation and, thus, melanocyte differentiation, suggesting that HMB45 is a melanocytic activation marker [14].

\section{Discussion}

Several researchers have used RBE to activate various types of cells [17-19]. Hagl et al. found that RBE, which contains oryzanols and tocopherol components, compensated agerelated mitochondrial dysfunction by increasing the mitochondrial respiration and membrane potential in a neurodegenerative mouse model. Choi et al. showed that the linoleic acid and $\gamma$-oryzanol components in RBE promoted hair growth by inducing hair follicles into the anagen stage (active growth phase) and increasing VEGF, IGF-1, and KGF expressions. Also, Fukumoto et al. reported that RBE had the ability to differentiate mesenchymal stem cells into osteogenic cells. These previous studies [17-19] were mainly focused on the organic compounds in rice bran extracts, such as vitamins, oryzanols, tocopherols, tocotrienols, and linoleic acid.

However, besides its organic compounds, rice bran is rich in minerals, such as $\mathrm{Si}, \mathrm{K}$, and $\mathrm{Mg}$. Liu et al. reported that rice 


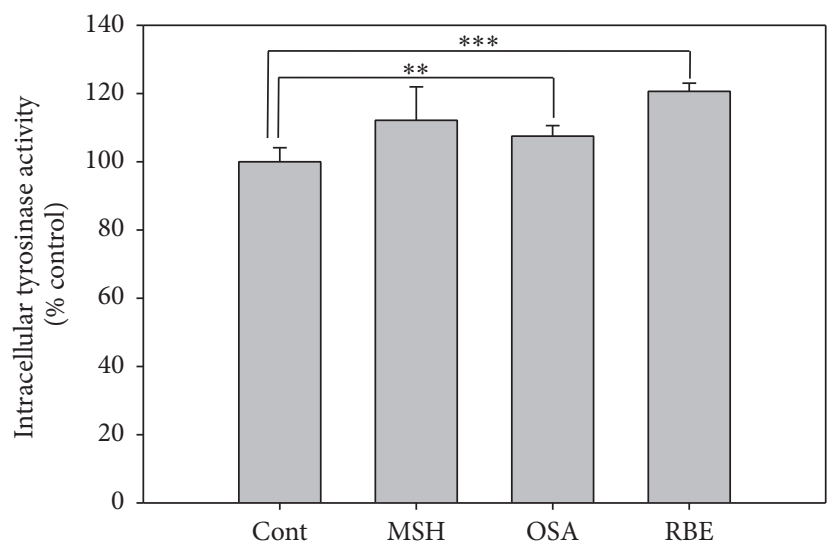

FIGURE 4: Representative graph of tyrosinase activity in melanocytes after 3 days cultured in differentiation media and after 3 days cultured in MSH, OSA, and RBE enriched media. Significant differences were determined by Student's $t$-test; ${ }^{* *} P<0.01 ;{ }^{* * *} P<0.005$.

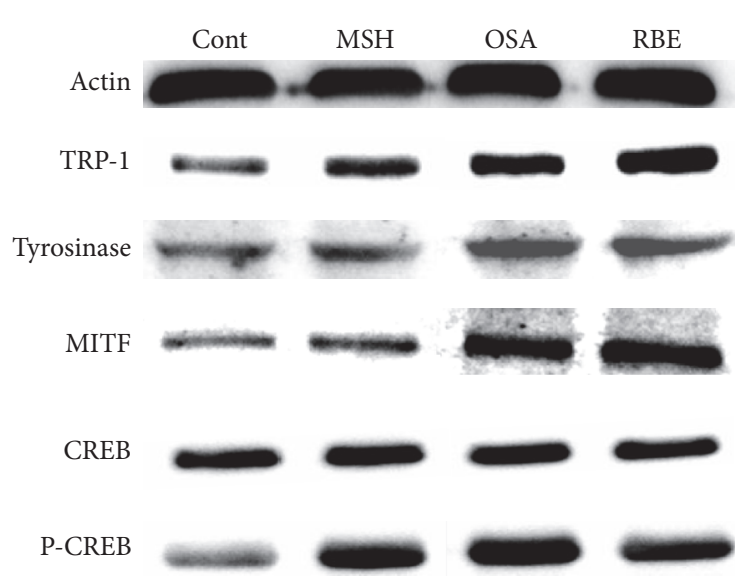

(a)

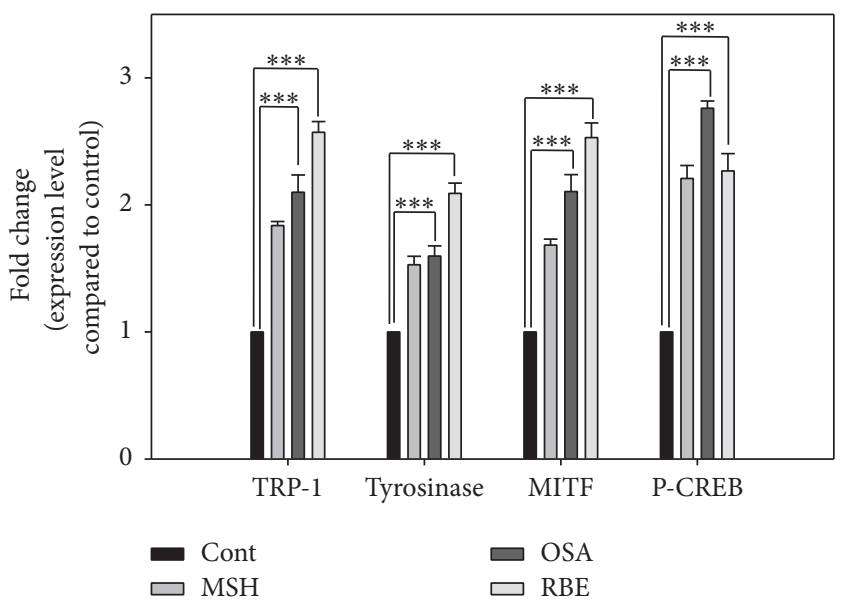

(b)

FIGURE 5: Melanogenesis effect in melanocytes after OSA and RBE were added and the cells cultured for 3 days. TRP-1, tyrosinase, MITF, and P-CREB increased remarkably compared to the control group. Significant differences were determined by Student's $t$-test; ${ }^{* * *} P<0.005$.

bran ash contains various mineral components, $\mathrm{Si}, \mathrm{C}, \mathrm{K}_{2} \mathrm{O}$, $\mathrm{CaO}, \mathrm{Na}_{2} \mathrm{O}, \mathrm{MgO}, \mathrm{Al}_{2} \mathrm{O}_{3}, \mathrm{ZnO}, \mathrm{MnO}_{2}$, and $\mathrm{Fe}_{2} \mathrm{O}_{3}$, with $\mathrm{Si}$ accounting for $65 \mathrm{wt} \%$ [20]. Hence, this study focused on RBE minerals, in particular silicic acid. The composition of RBE (Table 1) was similar to that of the rice bran ash studied by Liu et al. [20], suggesting that $30 \mu \mathrm{g} / \mathrm{mL}$ of RBE contained $5 \mu \mathrm{g} / \mathrm{mL}$ of soluble Si.

Silicate usually exists as $\mathrm{SiO}_{2}$, which has shown cytotoxicity in human cell lines (A431 and A549) [4]. Furthermore, in a rat liver cell line, $\mathrm{SiO}_{2}$ decreased cell viability in a dose-dependent manner [5]. In this study, there were no signs of apoptosis or cell damage on melanocytes (Figure 1) at $0.24 \mu \mathrm{g} / \mathrm{mL}$ OSA and $30 \mu \mathrm{g} / \mathrm{mL}$ RBE. Melanocytes were apparently normal in terms of morphology. It was, however, shown that they were stressed a little in the test of $\mathrm{LDH}$ activity.

Several studies have investigated the effects of OSA, which is less cytotoxic than silicate. For example, Dong et al. found that OSA significantly increased type 1 collagen and osteocalcin in the osteoblast-like cell lines MG-63 and U2-OS [7]. Similarly, Reffitt et al. showed increased type 1 collagen, alkaline phosphatase, and osteocalcin synthesis in osteoblastlike cell lines, MG-63 and $\mathrm{HCCl}$ [6]. Also, Grotheer et al. showed that OSA-releasing hydrogel decreased the inflammatory response and aided chronic wound recovery by inhibiting NF- $\kappa \mathrm{B}$ expression or enhancing $\mathrm{I} \kappa \mathrm{B}, \mathrm{NF}-\kappa \mathrm{B}-$ inhibiting protein [11].

In this study, we investigated the effect of OSA and RBE mineral components on melanocytes. OSA and RBE increased tyrosinase activity and melanin content, and both materials activated TRP-1, tyrosinase, MITF, and P-CREB in melanocytes.

Melanin synthesis increased in MSH, OSA, and RBE groups compared to the control group. Hence, melanin contents were elevated in MSH, OSA, and RBE groups (Figure 3) and Fontana-Masson staining confirmed that melanin was present in the melanocytes (Figure 6). These results suggest that OSA and RBE were able to stimulate the melanocytes 


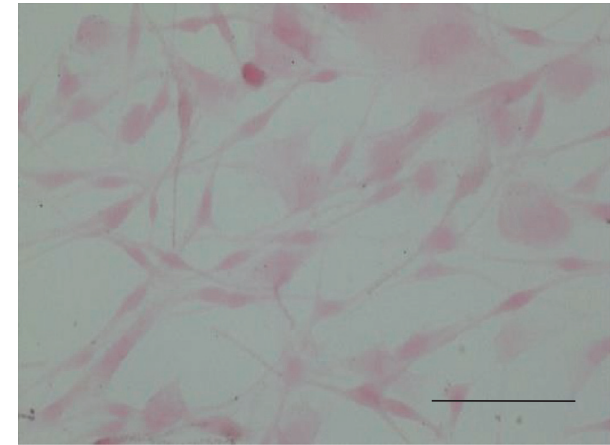

(a)

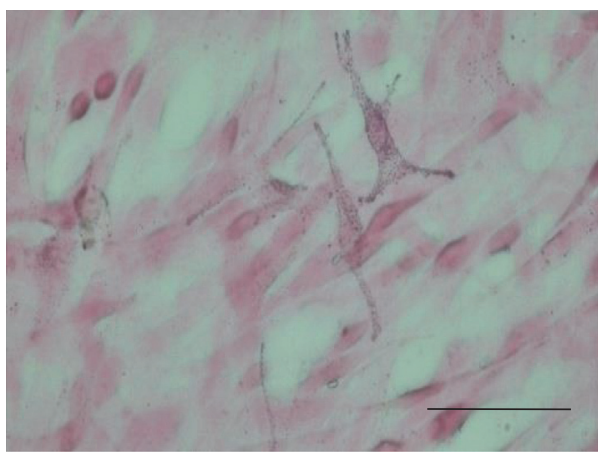

(c)

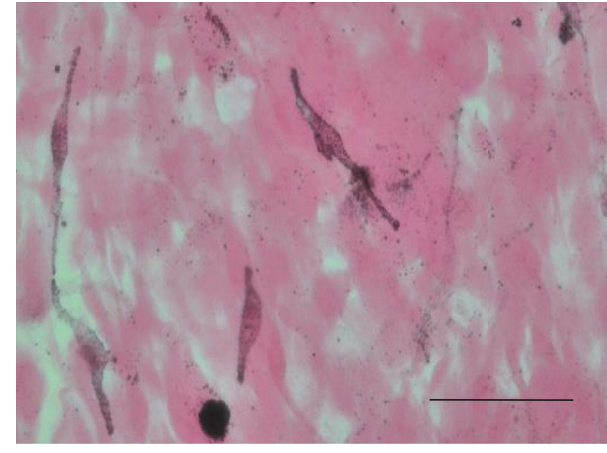

(b)

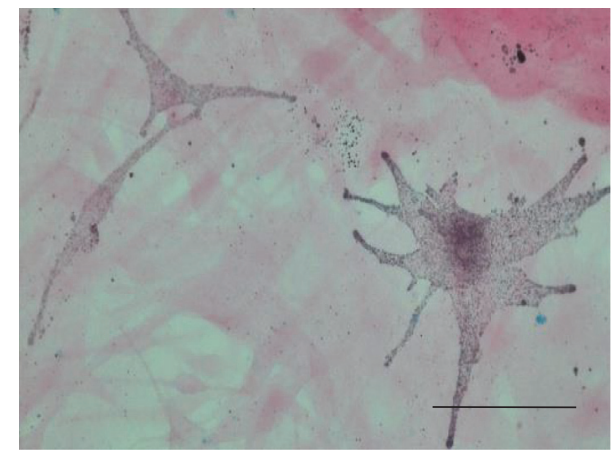

(d)

Figure 6: Representative images of Fontana-Masson stained melanocytes after 3 days cultured in differentiation media (dark color indicates secreted melanin). (a) Control, (b) $10 \mathrm{nMSH}$, (c) $2.4 \mu \mathrm{g} / \mathrm{mL}$ OSA, and (d) $30 \mu \mathrm{g} / \mathrm{mL}$ RBE. Original magnification: (a) $-(\mathrm{d}) \times 400$; scale bar: $50 \mu \mathrm{m}$.

TABLE 1: Chemical composition of RBE.

\begin{tabular}{lcccccc}
\hline Element & $\mathrm{Si}$ & $\mathrm{Ca}$ & $\mathrm{Mg}$ & $\mathrm{K}$ & $\mathrm{Na}$ & $\mathrm{P}$ \\
\hline $\mathrm{mg} / \mathrm{kg}$ (solution) & 180 & 5 & 17 & 2930 & 113 & 300 \\
\hline
\end{tabular}

TABLE 2: Results of staining after OSA and RBE treatment on melanocyte.

\begin{tabular}{lcccc}
\hline & Cont & MSH & OSA & RBE \\
\hline FM stain & - & ++ & + & + \\
HMB45 & + & ++++ & +++ & +++ \\
\hline
\end{tabular}

to synthesize melanin. Moreover, OSA and RBE groups had 10 and $20 \%$ higher tyrosinase activity than control group. Tyrosinase catalyzes melanin synthesis by oxidizing tyrosine to DOPA and the dehydrogenation of DOPA to dopaquinone [21]. In this study, RBE was more efficient at catalyzing melanin synthesis than MSH.

The Western blotting results showed that TRP-1, tyrosinase, and MITF expression levels were higher in the OSA and RBE groups compared to the control group (Figure 5). TRP1 oxidizes 5,6-dihydroxyindole-2-carboxylic acid (DHICA) to a carboxylated indole-quinone [22], which is one of the processes in melanin synthesis. Furthermore, MITF induces upregulation of the tyrosinase gene family [23]. The increase in MITF is associated with the HMB45 immunostaining results. This is because PMEL is involved in the deposition of melanin onto the internal fibrils of the melanosome, HMB45 staining is localized in melanosomes, and PMEL expression is transcriptionally regulated by MITF [16]. Hence, HMB45 can be used as a melanogenesis marker in melanocytes [16]. Thus, the positive HMB45 staining in OSA and RBE groups is due to the increased level of MITF. In addition, increased MITF expression level can be explained by the increased P-CREB level. Bu et al. showed that inhibition of CREB phosphorylation consequently decreased MITF expression in a B16-F10 melanoma cell line [24].

\section{Conclusion}

Melanogenesis is based on a complicated mechanism regulated by multiple factors. Among them, TRP-1, tyrosinase, and MITF are regarded as key melanogenic factors. In this study, we showed that OSA has the potential to promote melanogenesis and both RBE and OSA have similar cell viability, protein expression, and immunostaining results, suggesting that RBE comprises specific minerals which promote melanin synthesis through MITF and CREB phosphorylation. Therefore, RBE could be used as a novel material to promote melanin synthesis by stimulating melanocytes with its soluble $\mathrm{Si}$ and mineral components. 


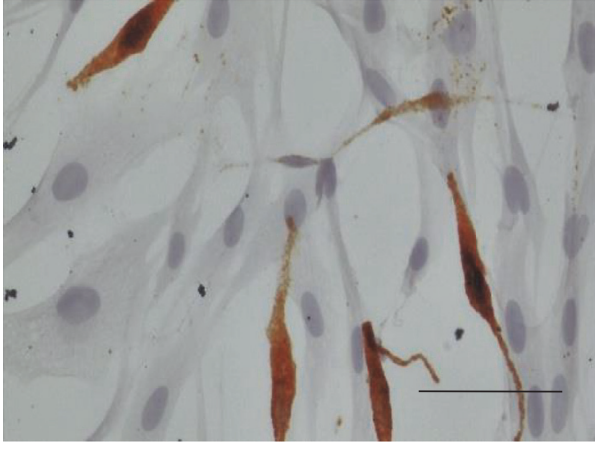

(a)

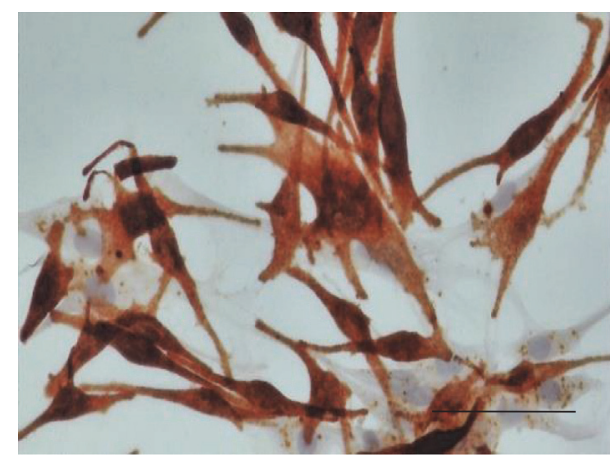

(c)

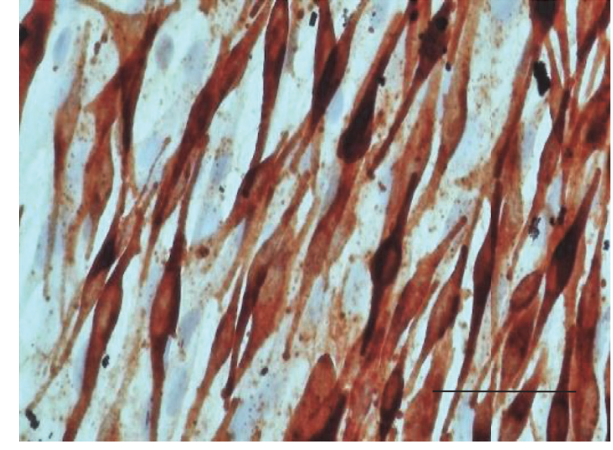

(b)

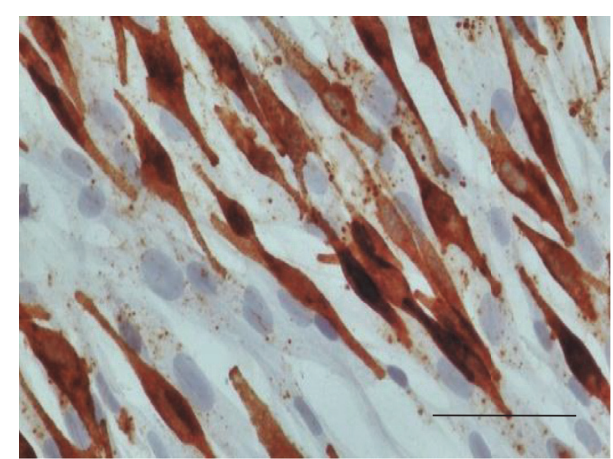

(d)

FIGURE 7: Representative images of HMB-45 immunostained melanocytes after 3 days cultured in differentiation media (brown color indicates melanosome). (a) Control, (b) $10 \mathrm{nMSH}$, (c) $2.4 \mu \mathrm{g} / \mathrm{mL}$ OSA, and (d) $30 \mu \mathrm{g} / \mathrm{mL}$ RBE. Original magnification: (a) $-($ d) $\times 400$; scale bar: $50 \mu \mathrm{m}$.

\section{Competing Interests}

The authors declare that there are no competing interests regarding the publication of this paper.

\section{Acknowledgments}

This study was supported by a grant of the Korean Health Technology R\&D Project, Ministry of Health \& Welfare, Republic of Korea (HN14C0086).

\section{References}

[1] S. H. Hong, M. J. Sim, and Y. C. Kim, "Melanogenesis-promoting effects of rhynchosia nulubilis and rhynchosia volubilis ethanol extracts in melan-a cells," Toxicological Research, vol. 32, no. 2, pp. 141-147, 2016.

[2] Y. A. An, J. Y. Hwang, J. S. Lee, and Y. C. Kim, "Cornus officinalis methanol extract upregulates melanogenesis in melan-a cells," Toxicological Research, vol. 31, no. 2, pp. 165-172, 2015.

[3] J. Park, H. Chung, S. H. Bang et al., "(E)-4-(3,4-dimethoxyphenyl)but-3-en-1-ol enhances melanogenesis through increasing upstream stimulating factor-1-mediated tyrosinase expression," PLoS ONE, vol. 10, no. 11, Article ID e0141988, 2015.

[4] M. Ahamed, "Silica nanoparticles-induced cytotoxicity, oxidative stress and apoptosis in cultured A431 and A549 cells," Human and Experimental Toxicology, vol. 32, no. 2, pp. 186-195, 2013.
[5] Y. Xue, Q. Chen, T. Ding, and J. Sun, " $\mathrm{SiO}_{2}$ nanoparticleinduced impairment of mitochondrial energy metabolism in hepatocytes directly and through a Kupffer cell-mediated pathway in vitro," International Journal of Nanomedicine, vol. 9, no. 1, pp. 2891-2903, 2014.

[6] D. M. Reffitt, N. Ogston, R. Jugdaohsingh et al., "Orthosilicic acid stimulates collagen type 1 synthesis and osteoblastic differentiation in human osteoblast-like cells in vitro," Bone, vol. 32, no. 2, pp. 127-135, 2003.

[7] M. Dong, G. Jiao, H. Liu et al., "Biological silicon stimulates collagen type 1 and osteocalcin synthesis in human osteoblastlike cells through the BMP-2/Smad/RUNX2 signaling pathway," Biological Trace Element Research, vol. 173, no. 2, pp. 306-315, 2016.

[8] M. Calomme, P. Geusens, N. Demeester et al., "Partial prevention of long-term femoral bone loss in aged ovariectomized rats supplemented with choline-stabilized orthosilicic acid," Calcified Tissue International, vol. 78, no. 4, pp. 227-232, 2006.

[9] T. D. Spector, M. R. Calomme, S. H. Anderson et al., "Cholinestabilized orthosilicic acid supplementation as an adjunct to calcium/vitamin D3 stimulates markers of bone formation in osteopenic females: a randomized, placebo-controlled trial," BMC Musculoskeletal Disorders, vol. 9, article 85, 2008.

[10] M. R. Calomme and D. A. Vanden Berghe, "Supplementation of calves with stabilized orthosilicic acid: effect on the $\mathrm{Si}, \mathrm{Ca}, \mathrm{Mg}$, and $\mathrm{P}$ concentrations in serum and the collagen concentration in skin and cartilage," Biological Trace Element Research, vol. 56, no. 2, pp. 153-165, 1997. 
[11] V. Grotheer, M. Goergens, P. C. Fuchs et al., "The performance of an orthosilicic acid-releasing silica gel fiber fleece in wound healing," Biomaterials, vol. 34, no. 30, pp. 7314-7327, 2013.

[12] A. Barel, M. Calomme, A. Timchenko et al., "Effect of oral intake of choline-stabilized orthosilicic acid on skin, nails and hair in women with photodamaged skin," Archives of Dermatological Research, vol. 297, no. 4, pp. 147-153, 2005.

[13] M. A. Hamid, M. R. Sarmidi, and C. S. Park, "Mangosteen leaf extract increases melanogenesis in B16F1 melanoma cells by stimulating tyrosinase activity in vitro and by up-regulating tyrosinase gene expression," International Journal of Molecular Medicine, vol. 29, no. 2, pp. 209-217, 2012.

[14] S.-Y. Chung, Y.-K. Seo, J.-M. Park et al., "Fermented rice bran downregulates MITF expression and leads to inhibition of $\alpha$ MSH-induced melanogenesis in B16F1 melanoma," Bioscience, Biotechnology and Biochemistry, vol. 73, no. 8, pp. 1704-1710, 2009.

[15] A. Kubanov, D. Proshutinskaia, V. Volnukhin, O. Katunina, and T. Abramova, "Immunohistochemical analysis of melanocyte content in different zones of vitiligo lesions using the MelanA marker," Acta Dermatovenerologica Alpina, Pannonica, et Adriatica, vol. 25, no. 1, pp. 5-9, 2016.

[16] J. Du, A. J. Miller, H. R. Widlund, M. A. Horstmann, S. Ramaswamy, and D. E. Fisher, "MLANA/MART1 and SILV/PMEL17/GP100 are transcriptionally regulated by MITF in melanocytes and melanoma," American Journal of Pathology, vol. 163, no. 1, pp. 333-343, 2003.

[17] K. Fukumoto, T. Tsuno, M. Taniguchi, and S. Terada, "Rice bran extract affects differentiation of mesenchymal stem cells potency into osteogenic cells," Cytotechnology, vol. 65, no. 6, pp. 937-943, 2013.

[18] J.-S. Choi, M.-H. Jeon, W.-S. Moon et al., "In vivo hair growthpromoting effect of rice bran extract prepared by supercritical carbon dioxide fluid," Biological and Pharmaceutical Bulletin, vol. 37, no. 1, pp. 44-53, 2014.

[19] S. Hagl, R. Grewal, I. Ciobanu et al., "Rice bran extract compensates mitochondrial dysfunction in a cellular model of early Alzheimer's disease," Journal of Alzheimer's Disease, vol. 43, no. 3, pp. 927-938, 2015.

[20] Y. Liu, Y. Guo, Y. Zhu et al., "A sustainable route for the preparation of activated carbon and silica from rice husk ash," Journal of Hazardous Materials, vol. 186, no. 2-3, pp. 1314-1319, 2011.

[21] A. Korner and J. Pawelek, "Mammalian tyrosinase catalyzes three reactions in the biosynthesis of melanin," Science, vol. 217, no. 4565, pp. 1163-1165, 1982.

[22] T. Kobayashi, K. Urabe, A. Winder et al., "Tyrosinase related protein 1 (TRP1) functions as a DHICA oxidase in melanin biosynthesis," The EMBO Journal, vol. 13, no. 24, pp. 5818-5825, 1994.

[23] C. Levy, M. Khaled, and D. E. Fisher, "MITF: master regulator of melanocyte development and melanoma oncogene," Trends in Molecular Medicine, vol. 12, no. 9, pp. 406-414, 2006.

[24] J. Bu, P.-C. Ma, Z.-Q. Chen et al., "Inhibition of MITF and tyrosinase by paeonol-stimulated JNK/SAPK to reduction of phosphorylated CREB," The American Journal of Chinese Medicine, vol. 36, no. 2, pp. 245-263, 2008. 


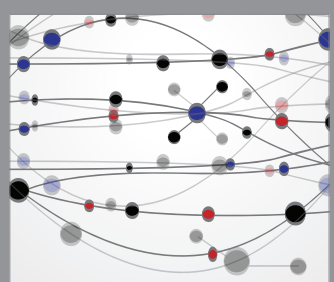

The Scientific World Journal
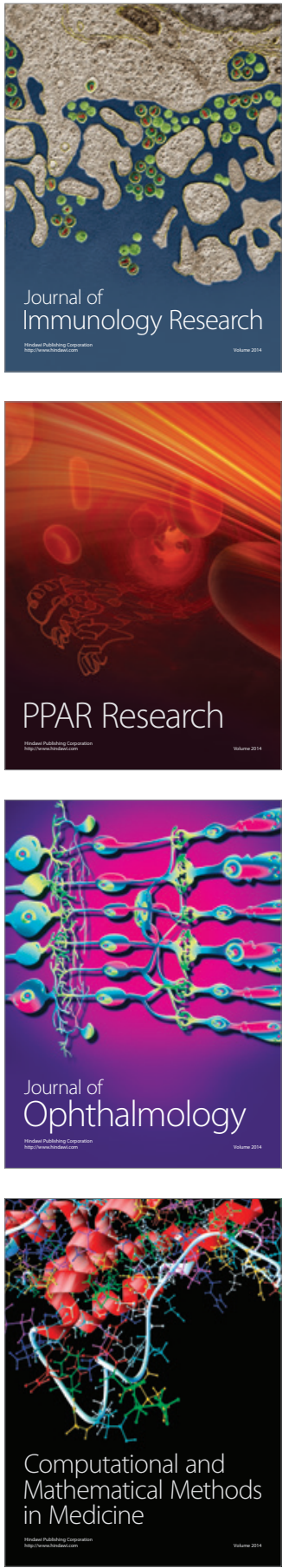

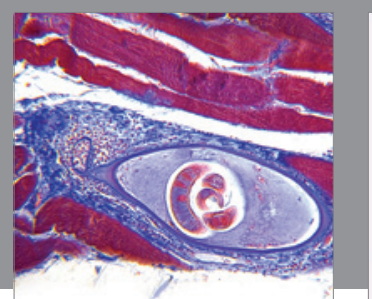

Gastroenterology Research and Practice

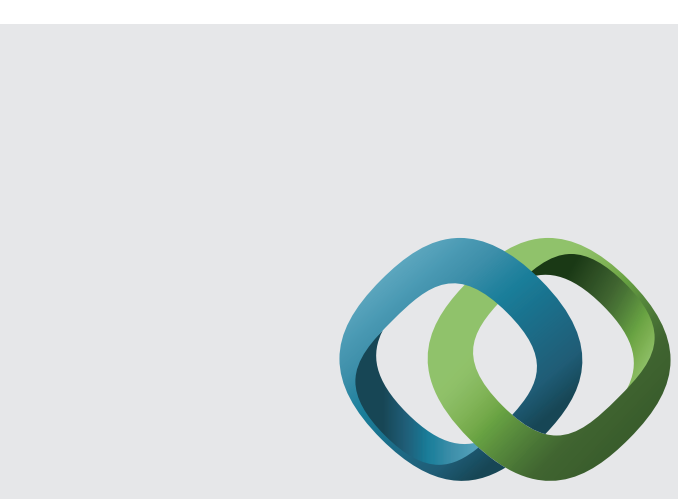

\section{Hindawi}

Submit your manuscripts at

http://www.hindawi.com
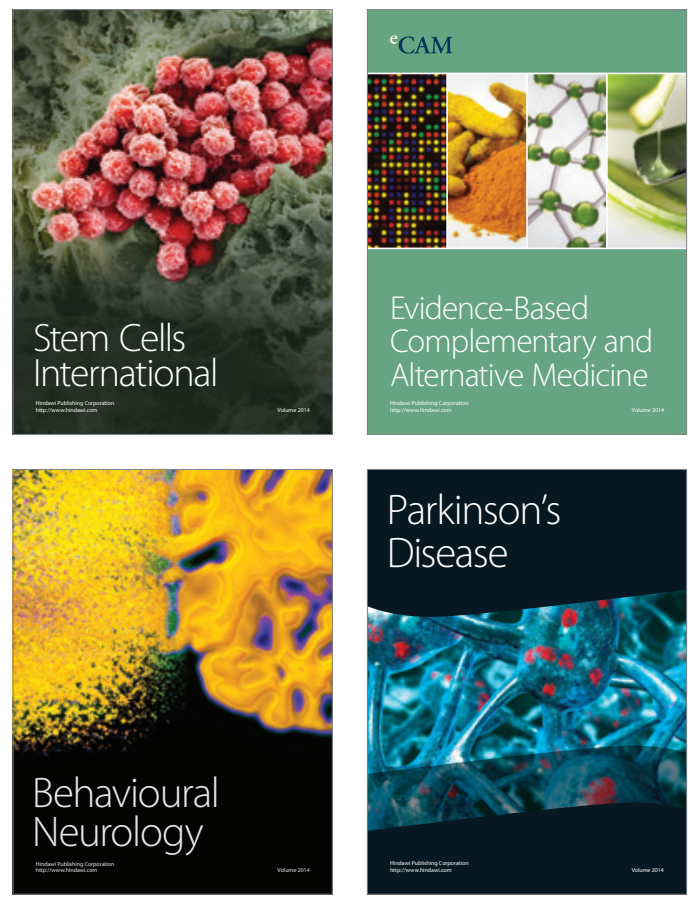
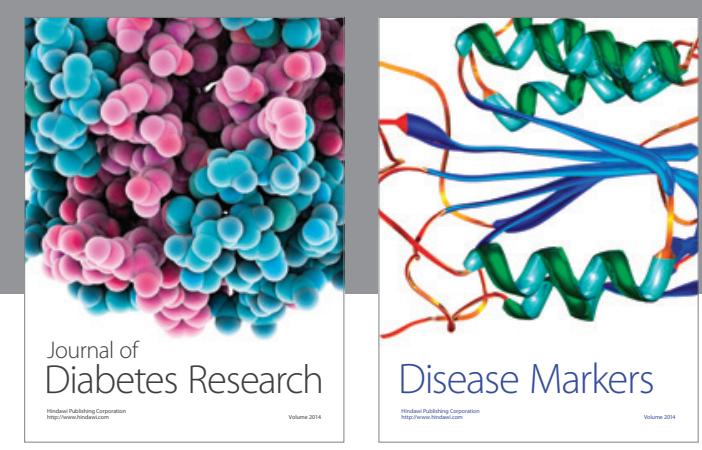

Disease Markers
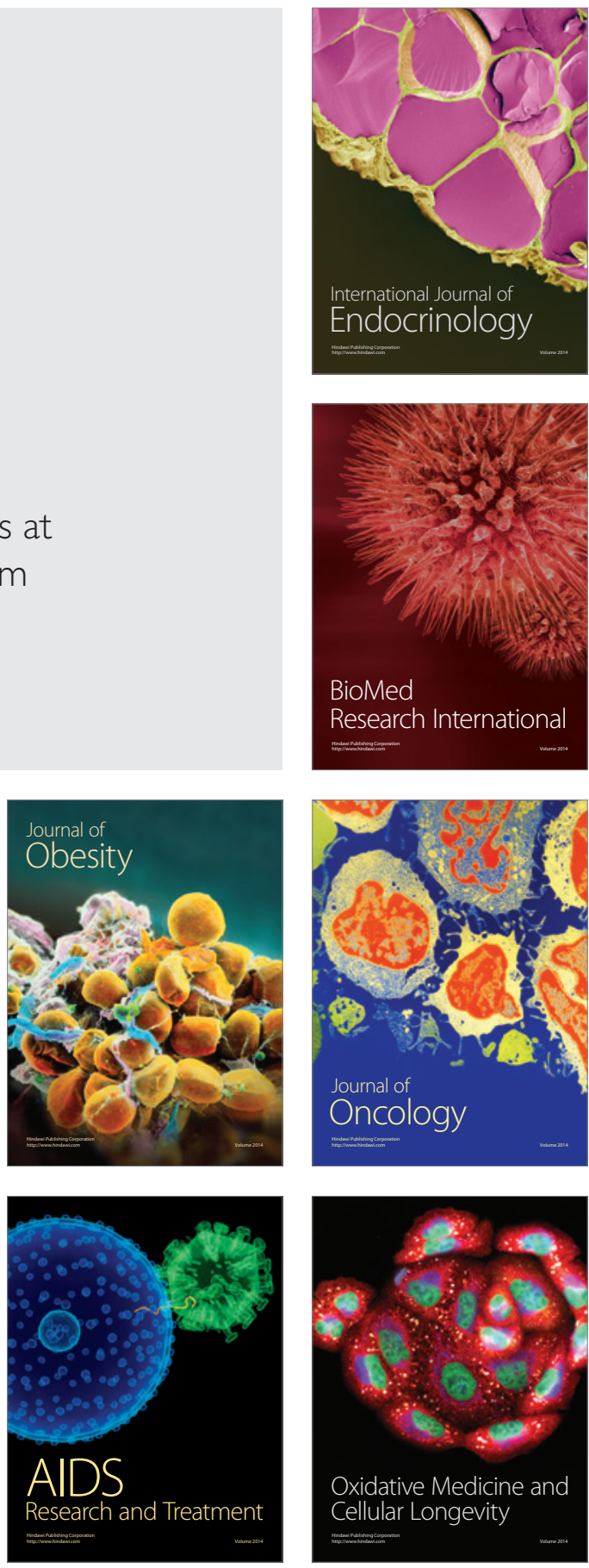